\title{
Berichtigung zu meiner Arbeit "Ober die Bewegung eines kleinen starren Probekörpers in beliebig vorgegebenen Gravitationsfeldern".
}

Von

G. Thomsen in Hamburg.

In meiner im Band 29 (1928) erschienenen Arbeit ist ein Fehler unterlaufen, der zwar für die sich dort anschließenden weiteren Untersuchungen keine Bedeutung hat, dessen Richtigstellung aber an und für sich von Interesse sein dürfte. Ich wurde von verschiedenen Seiten, zuerst von Herrn A. Wundheiler aus Warschau auf diesen Fehler aufmerksam gemacht.

Hat man im Riemannschen Raum eine Kurve $C$ in der Parameterdarstellung $x^{i}(\sigma)$, wo $\sigma$ die Bogenlänge ist, so kann man einen kontravarianten Vektor $u^{i}$ nach verschiedenen invarianten Gesetzen längs der Kurve $C$ verschieben. Gilt für die Schar der Vektoren $u^{i}(\sigma)$ längs $C$ :

$$
\frac{d u^{i}}{d \sigma}+\Gamma_{k l}^{i} u^{k} \frac{d x^{\prime}}{d \sigma}=0
$$

so hat man die Parallelverschiebung von Levi-Civita, gilt statt dessen

$$
\frac{d u^{t}}{d \sigma}+\Gamma_{k l}^{i} u^{k} \frac{d x^{3}}{d \sigma}=-\left[g_{r t} u^{r}\left(\frac{d^{2} x^{k}}{d \sigma^{z}}+\Gamma_{p q}^{t} \frac{d x^{p}}{d \sigma} \frac{d x^{q}}{d \sigma}\right)\right] \cdot \frac{d x^{\prime}}{d \sigma},
$$

so hat man die sogenannte Verschiebung von Fermi, gilt endlich das Gesetz (3) $\frac{d u^{i}}{d \sigma}+\Gamma_{k l}^{i} u^{k} \frac{d x^{\prime}}{d \sigma}=-\left[g_{r l} u^{r}\left(\frac{d^{2} x^{2}}{d \sigma^{8}}+\Gamma_{p q}^{t} \frac{d x^{p}}{d \sigma} \frac{d x^{q}}{d \sigma}\right)\right] \cdot\left(\frac{1}{\sin ^{2} \theta} \frac{d x^{i}}{d \sigma}-\frac{\cos \theta}{l \cdot \sin ^{2} \theta} u^{i}\right)$, wobei

$$
l=\sqrt{g_{i k} u^{i} u^{k}} \quad \text { und } \quad \cos \theta=\frac{g_{k} u^{u^{d}} \frac{d x^{k}}{l}}{l}
$$

gesetzt ist, so hat man die Verschiebung von Wundheiler.

Die Verschiebung von Wundheiler ist durch folgende drei geometrischen Eigenschaften gekennzeichnet: 
640. G. Thomsen. Uber die Bewegung eines kleines starren Probekörpers.

A. Verschiebt man mittels dieses Gesetzes den Vektor $u^{i}$ vom Punkte $P$ der Kurve $C$ nach dem Nachbarpunkt $P+d P$ anf $C$, so bildet der dort entstehende Vektor $u^{i}+\Delta u^{i}$ in $P+d P$ mit der Kurve $C$ denselben Winkel $\Theta$ wie $u^{i}$ in $P$.

B. $u^{i}+\Delta u^{i}$ hat dieselbe "Länges $l$ wie $u^{i}$.

C. $u^{i}+\Delta u^{i}$ liegt in dem Flächenelement, das in $P+d P$ bestimmt ist durch die Tangente von $C$ und denjenigen Vektor, der aus $u^{i}$ durch die Verschiebung (1) von Levi-Civita hervorgeht.

Auf Seite 105 meiner Arbeit ist irrtümlich die Behauptung aufgestellt worden, dab die Verschiebung (2) von Fermi die Eigenschaften A., B., C. besitzt. Dies ist offenbar nur für zur Kurve $C$ senkrechte Vektoren $u^{i}$ richtig, bei denen wegen $\Theta=\pi: 2$ die Verschiebung von Wundheiler mit der von Fermi zusammenfällt. Da es sich bei dem mechanischen Problem meiner Arbeit nur um zur Kurve senkrechte Vektoren handelt, bleiben die im Späteren abgeleiteten Formeln richtig.

(Fingegangen am 4. Mai 1929.) 